University of Nebraska - Lincoln

DigitalCommons@University of Nebraska - Lincoln

$10-28-2021$

\title{
Flock-species richness influences node importance and modularity in mixed-species flock networks
}

\author{
Priti Bangal \\ Centre for Ecological Sciences, Indian Institute of Science, pritibangal@gmail.com \\ Hari Sridhar \\ Centre for Ecological Sciences, Indian Institute of Science \\ Daizaburo Shizuka \\ University of Nebraska-Lincoln, dshizuka2@unl.edu \\ Laura N. Vander Meiden \\ University of Nebraska - Lincoln, I.vandermeiden@huskers.unl.edu \\ Kartik Shankar \\ Centre for Ecological Sciences, Indian Institute of Science, kshanker@gmail.com
}

Follow this and additional works at: https://digitalcommons.unl.edu/bioscibehavior

Part of the Ecology and Evolutionary Biology Commons

\begin{abstract}
Bangal, Priti; Sridhar, Hari; Shizuka, Daizaburo; Vander Meiden, Laura N.; and Shankar, Kartik, "Flockspecies richness influences node importance and modularity in mixed-species flock networks" (2021). Papers in Behavior and Biological Sciences. 85.

https://digitalcommons.unl.edu/bioscibehavior/85
\end{abstract}

This Article is brought to you for free and open access by the Papers in the Biological Sciences at DigitalCommons@University of Nebraska - Lincoln. It has been accepted for inclusion in Papers in Behavior and Biological Sciences by an authorized administrator of DigitalCommons@University of Nebraska - Lincoln. 


\title{
Flock-species richness influences node importance and modularity in mixed-species flock networks
}

\author{
Priti Bangal, ${ }^{1,4}$ Hari Sridhar,, ${ }^{1,2}$ Daizaburo Shizuka, ${ }^{3}$ \\ Laura N. Vander Meiden, ${ }^{3}$ \& Kartik Shanker ${ }^{1}$ \\ 1 Centre for Ecological Sciences, Indian Institute of Science, Bengaluru 560012, \\ India \\ 2 National Centre for Biological Sciences, Bengaluru 560065, Karnataka, India \\ 3 School of Biological Sciences, University of Nebraska-Lincoln, 1104 T Street, \\ Lincoln, NE 68588-0118, USA \\ 4 Present Address: Nature Conservation Foundation, Mysore, Karnataka 570017, \\ India \\ Correspondence - Priti Bangal, pritibangal@gmail.com
}

\begin{abstract}
Interdependencies in social groups of animals are a combination of multiple pairwise interactions. Heterospecific groups are often characterized by important species that contribute more to group initiation, maintenance or function than other species. However, in large heterospecific groups, many pairwise interactions are not realized, while others may not be biologically significant, confounding inferences about species importance. Hence, in this study, we examine context dependent changes in species importance and assortment in mixed-species bird flocks from a tropical field site in Southern India using social network analysis. Specifically, we ask how the structural importance of a species and the clustering patterns
\end{abstract}

Published in Oecologia (2021)

https://doi.org/10.1007/s00442-021-05053-z

Copyright (C) 2021 by the Authors, under exclusive license to Springer-Verlag GmbH

Germany, part of Springer Nature. Used by permission.

Submitted 24 May 2020; accepted 28 September 2021; published 28 October 2021. 
of species relationships depends on species richness in mixed-species flocks. We constructed both raw and filtered networks; while our results are largely correlated, we believe that filtered networks can provide insights into community-level importance of species in mixed-flocks while raw networks depict flock-level patterns. We find significant differences in flocks of different richness in that different species emerge as structurally important across flocks of varying richness. We also find that assortment is higher in two-species flocks and decreases with an increase in the number of species in the flock ('flock richness' hereafter). We argue that the link between structural importance of species in mixed-species flock networks and their functional significance in the community critically depends on the social context: namely, the species richness of the mixed-species flock. We propose that examining species structural importance at different flock-richness values provides insights into biologically meaningful functional roles of species. More generally, we suggest that it is important to consider context when interpreting species centrality and importance in network structure.

Keywords: Mixed-species, Social networks, Co-occurrence networks, Functional importance, Structural importance

\section{Introduction}

Mixed-species social groups are important associations that serve different functions across animal communities. The adaptive advantages for grouping are often context dependent in mixed-species social groups (Goodale et al. 2017). Such groups are commonly seen in several taxa such as ungulates, primates, reef fish, cetaceans and birds (see Stensland et al. 2003 for review of mammal groups, Lukoschek and McCormick 2000 for fish, Greenberg 2000 for birds, and Goodale et al. 2017 for a summary). Protection against predators and increased foraging are two widely discussed benefits of such groups. Group size benefits (or dilution) may be more prominent in some groups (Beauchamp 2013), whereas in other mixed-species social groups, benefits from eavesdropping on social information about predators may be the primary anti-predatory benefit (Magrath et al. 2009). Similarly, foraging benefits may also be accrued either by copying foraging locations (Krebs 1973), decreased time invested in vigilance or more directly by following individuals that make resources available to other species as a byproduct of their active behavior (Giraldeau and Beauchamp 1999). Very often, multiple mechanisms operate within the same group and it is often difficult to isolate a single reason for grouping (Goodale et al. 2020). 
Mixed-species bird flocks are heterospecific groups of two or more species that forage and move together. The main benefits that species gain from participating in these flocks are related to improved foraging and defense mechanisms against predators (Greenberg 2000; Sridhar et al. 2009). These benefits may be gained via pairwise interactions (e.g. a sallyer benefits from following gleaners that flush out insects from the understory) or group-level mechanisms such as eavesdropping on alarm calls of other group members, the dilution effect, and the many eyes effects of improved predator detection or improved food finding. Some species that participate in mixed-species bird flocks (referred to as flocks, hereafter) are obligate flock participants and depend solely on flocks for foraging (Jullien and Clobert 2000; Munn and Terborgh 1979). However, benefits to all participants in flocks are not equal given that these species show variation in phenotypic and ecological traits and behavior. Typically, in mixed-flocks, multiple pairwise interactions between species are possible, but are not always realized. Many species present in mixed-species flocks may contribute only to the overall group size of the flock, and benefit provision and gain may be asymmetric between species pairs (Harrison and Whitehouse 2011; Sridhar and Shanker 2014). Hence, flocks can therefore create opportunities for bystanders, which are commensals at the flock level, and do not have specific pairwise species associations with other participants.

Many mixed-species flocks have dynamic membership of both individuals and species. Interactions in mixed-species bird flocks occur at an individual level and can occur between both conspecifics and heterospecifics, whereas we refer to associations as emergent population level interdependencies. Interactions between individuals of different species at the flock level create a network of associations at the population level where species rather than individuals are connected. Viewing these dependencies at the species level in a network framework can help reveal patterns in these communities, such as key species that play a role in the assembly of these communities, the ecological drivers of mixed-species flocks, and potentially, factors that maintain stability of this network (e.g. Marthy and Farine 2018; Sridhar et al. 2013). This approach is also useful in examining species interactions beyond a pairwise dyadic interaction level and to potentially examine how these interactions scale up when examining the system as a 
whole (Vasas and Jordán 2006). Using individuals as nodes can help in understanding social group-level dependencies and variation across individuals (Farine et al. 2012; Farine and Milburn 2013).

Social network analysis has been used to identify central nodes (or species) in flock co-occurrence networks (Marthy and Farine 2018; Sridhar et al. 2013). The species that emerge as important nodes and belong to the same trophic level have been referred to as within-trophic keystones. Their removal from the network could break down several interactions in the network and affect the ecology of mixed-species flocks (Marthy and Farine 2018). Centrality values of nodes from the networks from multi-species groups (such as degree or weighted degree) have been proposed and used to ascribe structural importance to different nodes in the network (Borah et al. 2018; Marthy and Farine 2018; Mokross et al. 2014; Sridhar et al. 2013). However, flock networks are largely 'gambit of the group' networks. Gambit of the group networks are based purely on co-occurrences of species in flocks rather than on direct interactions (Croft et al. 2008; Franks et al. 2010; Whitehead and Dufault 1999). In such networks, two species which are represented by nodes in the network, are connected to each other if these species occur in the same flock irrespective of direct interactions between the individuals. Therefore, centrality measures might not always be reliable indicators of species functional importance in interactions. A major goal of this study is to determine whether we can disentangle meaningful species roles from flock association data.

Network analysis can also be used to detect internal substructures (e.g., tightly linked clusters of nodes) based on the interactions within them (Girvan and Newman 2002; Shizuka and Farine 2016). In mixedspecies flocks, species-specific interactions and microhabitat preferences may lead to sub-structures within the associations network (Borah et al. 2018). These sub-structures may point to meaningful biological interactions of functional importance. In this study, we examine networks of flocks with different number of species (referred to as flock species richness, hereafter) to make inferences about the relationship between structural and functional importance of species in flocks. We also examine network structure to understand the potential mechanisms of assembly as flocks increase in size. Flocks typically increase in size by addition of new species to a group; there is a positive relationship between flock richness and size (Goodale et al. 
2009). Species join flocks as entire family groups or pairs or solitary individuals and once a species has joined a flock, it is unlikely that a different family group/pair of the same species will join the same flock. Hence, we argue that flocks increase in size mostly through the addition of new species. We therefore use flock species richness as a representation of flock size in this study.

Earlier work suggests that flock assembly varies with flock species richness, with non-randomness in species associations decreasing from small to large flocks (Bangal et al. 2021). There is clear phenotypic assortment in small flocks as opposed to large flocks that appear to be random associations between species (Bangal et al. 2021). This may be particularly true of two-species flocks where there is a definite interaction between pairs (see Sridhar and Shanker 2014), as opposed to species pairs that are only found in flocks of many species. Moreover, these patterns of flock species richness may affect a species' position in the network in a way that does not reflect their ecological significance: e.g., a species that is found only in large flocks may have a large number of connections, but each of these may not be biologically meaningful. Thus, examining network sub-structures at different flock species richness classes can lead to insights into how species associations are created in flocks. From networks built from small flocks, we can understand how community structure is established initially and how links across structures are established. Examining these structural properties of networks emerging from increasing flock species richness can also demonstrate how these associations change as flocks grow larger.

In this study, we examine the influence of flock species richness on species importance in networks and assess substructures to understand how species assortment changes with increasing flock richness. Specifically, we ask the following questions: (1) How does the structural importance of species change across networks of increasing flock species richness?, (2) How do species assort in two-species flocks, which may provide the most insight into biologically significant pairwise interactions?, and (3) Do assortment patterns between species get diluted with increasing flock species richness? A general question of interest in our study is to examine whether the patterns of interest change continuously across the range of flock richness, or if there are abrupt shifts and higher-order effects. 


\section{Methods}

\section{Study area}

The fieldwork for this study was conducted in Anshi Range of the Kali Tiger Reserve, Karnataka, India. Our study sites were located on forest trails around Anshi Nature Camp (15.00 $\left.{ }^{\circ} \mathrm{N}, 74.39^{\circ} \mathrm{E}\right)$ and Anshi Village $\left(14.99^{\circ} \mathrm{N}, 74.37^{\circ} \mathrm{E}\right)$. Data was collected in the non-breeding season between the months of December and March. This is also the migratory season, when winter visitors migrate to our field site for the non-breeding season.

\section{Data collection}

We collected data on flock composition by actively searching for mixed-flocks on 12 unique forest trails. Every trail was about 3-5 kms long. The sampling locations for each trail were at least $250 \mathrm{~m}$ away from every other trail. Each trail was sampled once every 10 days during each field season. The minimum duration between two sampling sessions on the same trail was at least 7 days to ensure independence of flock formation events. Our sampling was restricted only to mixedspecies flocks and we did not include aggregations at clumped resources in our study. The habitat was uniform across trails and flocks across trails did not vary in composition. A foraging group of birds comprising of two or more species moving together for at least $5 \mathrm{~min}$ was called a mixed-species flock. An individual was said to be in the flock if it was within a $10 \mathrm{~m}$ radius from its nearest neighbor in the group. Once we encountered a flock, we followed it for $15 \mathrm{~min}$ and recorded all the species present in the flock that were detected visually and acoustically. Based on our previous experience of observing flocks in these areas (Sridhar et al. 2013) we stayed with each flock for 15 min to capture a snapshot of flock composition. Given the habitat structure and observer detection, $15 \mathrm{~min}$ is a long enough window to capture most species present in the flock without capturing a change in species composition due to new species joining or existing species leaving the flock during a single observation bout. 


\section{Dataset}

We used flock composition data from three years of field sampling (2010, 2011, and 2017) for this analysis. Since flocks sampled in different years were compositionally similar, we pooled data from different years for the analysis. Over the sampling period of three years, we observed 620 flocks in the study area. From our previous work in the study area, we know that flock composition from the same trail and area were not identical and flock formation occurred over small time scales (minutes to hours) at our study site (Sridhar et al. 2013). Since flocks in our study area are temporary and form and dissolve over small timescales, and exhibit turnover of species over small timescales, every flock formation event was considered to be independent across days and trails. A cluster analysis on flock composition in a previous study conducted at our study site showed that flocks from the same location do not cluster together in a dendrogram (Sridhar et al. 2013). Therefore, each flock record was considered an independent sample.

\section{Analysis}

We dropped species that occurred in less than $1 \%$ of the flocks (less than 6 flocks in our dataset), from the analysis to avoid using chance occurrences. We classified flocks into different richness classes2-species flocks, 3-5 species flocks, 6-10 species flocks and 11-22 species flocks. For each of these categories, we compared structural patterns of species networks based on flock associations in these different classes of species richness. To assess the robustness of our results, we also repeated the analyses with networks in which edges were filtered (details mentioned ahead) based on their statistical significance using a null model approach. In addition to providing evidence for robustness for the analysis, we also propose that both raw and filtered networks are indicative of different processes in flocks and flocking communities. The raw networks include 'all' associations in the networks and therefore encompass important, unimportant, and rare interactions in flocks, capturing the diversity of patterns of associations in every flock. On the other hand, the filtered networks filter out the non-significant interactions in flocks based on the frequency 
of co-occurrence of species in all the flocks. This therefore only retains the non-random associations and uses only the non-random edges to arrive at node importance therefore allowing us to evaluate nodes that may be important not just within flocks but also overall in bird communities. Thus, while the raw networks represent group-level patterns in mixed-species flocks, the filtered networks indicate community-level significance of species and interactions.

\section{Network construction}

In the raw networks, all species pairs that co-occurred in flocks were used to construct the network. The edges were weighed by the frequency of co-occurrence of species pairs, divided by the total number of flocks, in each flock-richness category.

In the filtered networks, the edges were filtered for significant associations based on an association index calculated for every species pair. The association index was calculated as the following:

$$
\text { Association index }=\frac{O-E}{\sigma}
$$

where $O$ is the observed co-occurrence of a species pair, $E$ is the expected co-occurrence of the pair and $\sigma$ is the standard deviation of the expected co-occurrence of the species. The expected co-occurrence was calculated from randomizations on the species by flock presence absence matrix.

Randomizations were set up in the following manner: Since we were interested in examining differences in flocks of different richness values, we kept the number of flocks in each richness class in our expected data equal to the number of flocks in the observed dataset. The observed data matrix was randomized by holding the column totals (flock richness) constant and using the species occurrences as proportions. For each randomized matrix, we calculated a co-occurrence value for every species pair. We performed 1000 iterations and calculated a mean co-occurrence value (E) for every species pair. The standard deviation around the mean of the 1000 iterations was used as $\sigma$. We filtered out all pairs with an association strength value of less than 1.96 to arrive at only significantly positively associated species pairs for the filtered networks. The randomizations were done on the 
whole species by flock matrix and each matrix was filtered into the corresponding flock-richness subsets to calculate the association index for species pairs in every flock-richness category.

\section{Network measures}

Weighted degree We used weighted degree, which is a commonly used measure of centrality in networks as the measure of species structural importance in flocks. Weighted degree is a node-based measure which is the sum of weights of all edges that pass through the node under consideration.

$$
\mathrm{WD}=\sum_{i=1}^{n} W i
$$

Here, WD is the weighted degree of the node also referred to as node strength, $W$ is the edge weight and $i$ is the number of edges that pass through the node for which weighted degree is being calculated.

We use unweighted networks for filtered associations. Hence, we use degree centrality as a measure of structural importance in this analysis. Therefore, there are multiple species with the same centrality values in this category.

Calculating modularity We ran a 'community detection algorithm' based on the Louvian method on the networks built using the methods described above. Clusters of closely connected nodes (often termed 'communities' in network parlance) were detected based on modularity optimization (Newman 2006). Modularity is the measure of separation between two clusters calculated based on the number of edges within versus across clusters. In this method, each vertex is assigned to a unique cluster and a modularity score is calculated. At each step, the vertices are reassigned to clusters and a network structure that gives the maximum modularity is calculated. This process is repeated until modularity cannot be increased further.

Comparing the structure of species associations across flockrichness using assortativity We measured how closely the modular structure of larger networks reflected the patterns of associations in 2-species flocks. We reason that species associations in 2-species 
flocks are more likely to reflect reliable and known interactions between species. Two-species flocks are the smallest subunits of mixedspecies flocks where at least one of the species is a benefit provider and the other, the receiver. We can use the measure of structure in these two-species flocks to determine the degree to which these interactions are retained in flocks with more species. We use the measure of assortativity to examine how these patterns of associations change in structure of the network as we build them from data on larger flocks. The nodes that do not appear in the two-species flock networks were dropped from this analysis. The assignments of species into clusters within two-species flock networks were considered as a discrete node attribute, and this was then used to measure the assortment coefficient using assortment.discrete() function in the $\mathrm{R}$ package 'assortnet' (Farine 2014; Newman 2002, 2003) in each network. This is a modified use of the measure of robustness of community assignments from Shizuka and Farine (2016).

We tested whether these patterns of assortment were different from those expected by chance using a null model approach. Our null model was based on node-label permutations in which the attribute of interest (here, the assignment of a node into different clusters in the 2-species networks using the Louvian community detection method described above) was randomly shuffled across nodes in the network (Weiss et al. 2021). We then recalculated the assortativity index in this randomized network. We conducted this procedure 1,00o times for each network and compared the empirical assortment coefficient against the 95\% confidence interval generated from the randomized networks.

All analyses were implemented in $R$ ( $R$ core team 2018) using the packages igraph, assortnet and EcoSimR.

\section{Results}

We recorded 620 flocks over three years of data. The flock richness ranged from 2 to 22 species. A total of 64 different species participated in flocks at least once in our study area. Of those, 42 species participated in more than 6 flocks. 


\section{Identifying important species in flocks of different richness}

\section{Raw networks}

We plotted the weighted degree of all the species, ranked in the decreasing order to identify different structurally important species for raw and filtered networks (Supplementary material: S1, S2 The weighted degree and degree values for both network types are provided in $\mathrm{S}_{3}$ and $\mathrm{S}_{4}$ ). We selected six species with highest weighted degree values in each network to compare structural importance across flocks of different richness. The top six species which we refer to as the core species usually stand out (except in 3-5 species flock networks) and have substantially higher weighted degree values as opposed to the other species when arranged in decreasing order of weighted degree. We found that the membership of this group changes with flock species richness (Table 1). The composition of the core species is similar in all-flock and large-flock (10-22 species) networks but is different in the smaller flock networks (2 species and 3-5 species).

\section{Filtered networks}

Overall, the membership of core species that emerged as important from the filtered network is only marginally different from the raw network (Table 1).

\section{Network clusters and assortment}

\section{Raw networks}

Five and seven communities were detected in the raw and filtered twospecies flock networks, respectively (Fig. 1A, B; Supplementary material S5, S6). Overall segregation between communities decreased with flock richness (Fig. 1; Table 2). We then examined the assortativity of networks of larger sizes using the communities detected in two-species flocks as functional groups. This assortativity index decreased as flock species richness increases, suggesting that these species relationships that are clear in small flocks become diluted in larger flocks. Assortativity is more positive than expected from randomized networks 
Table 1 Summary of species that emerge as structurally important in each flockrichness class for both raw and filtered networks

\begin{tabular}{|c|c|c|c|c|c|c|c|c|c|c|}
\hline \multirow{2}{*}{$\begin{array}{l}\text { Species names } \\
\text { Western-crowned Warbler } \\
\quad \text { (Phylloscopus occipitalis) }\end{array}$} & \multicolumn{2}{|c|}{$\begin{array}{c}2 \\
\text { species }\end{array}$} & \multicolumn{2}{|c|}{$\begin{array}{l}3-5 \\
\text { species }\end{array}$} & \multicolumn{2}{|c|}{$\begin{array}{c}\text { 6-10 } \\
\text { species }\end{array}$} & \multicolumn{2}{|c|}{$\begin{array}{l}10-22 \\
\text { species }\end{array}$} & \multicolumn{2}{|c|}{$\begin{array}{l}\text { All } \\
\text { Flocks }\end{array}$} \\
\hline & $X$ & $X$ & $\mathrm{X}$ & $\mathrm{X}$ & $\mathrm{X}$ & $\mathrm{X}$ & $\mathrm{X}$ & $\mathrm{X}$ & $\mathrm{X}$ & $\mathrm{X}$ \\
\hline $\begin{array}{l}\text { Greater racket-tailed Drongo } \\
\text { (Dicrurus paradiseus) }\end{array}$ & $X$ & & & $X$ & $\mathrm{X}$ & $\mathrm{X}$ & $X$ & $\mathrm{X}$ & $X$ & $\mathrm{X}$ \\
\hline $\begin{array}{l}\text { Malabar Woodshrike } \\
\qquad \text { (Tephrodornis sylvicola) }\end{array}$ & $\mathrm{X}$ & & $X$ & & $\mathrm{X}$ & & & & & \\
\hline $\begin{array}{l}\text { Dark-fronted Babbler } \\
\text { (Rhopocichla atriceps) }\end{array}$ & $X$ & $\mathrm{X}$ & & & & & & & & \\
\hline $\begin{array}{l}\text { Oriental White- eye } \\
\quad \text { (Zosterops palpebrosus) }\end{array}$ & $X$ & $X$ & & & & & & & & \\
\hline $\begin{array}{l}\text { Orange Minivet } \\
\qquad \text { (Pericrocotus flammeus) }\end{array}$ & $\mathrm{X}$ & $X$ & $X$ & & & & $X$ & $\mathrm{X}$ & $\mathrm{X}$ & $\mathrm{X}$ \\
\hline $\begin{array}{l}\text { Brown-cheeked Fulvetta } \\
\text { (Alcippe poioicephala) }\end{array}$ & & $X$ & & $X$ & & $\mathrm{X}$ & $X$ & $X$ & $\mathrm{X}$ & $\mathrm{X}$ \\
\hline $\begin{array}{l}\text { Ashy Drongo } \\
\quad \text { (Dicrurus leucophaeus) }\end{array}$ & & $\mathrm{X}$ & $\mathrm{X}$ & $\mathrm{X}$ & & & & & & \\
\hline $\begin{array}{l}\text { Yellow-browed Bulbul } \\
\text { (Acritillas indica) }\end{array}$ & $\mathrm{X}$ & & & $\mathrm{X}$ & & $\mathrm{X}$ & $X$ & $\mathrm{X}$ & $\mathrm{X}$ & $\mathrm{X}$ \\
\hline $\begin{array}{l}\text { Greenish Warbler } \\
\quad \text { (Phylloscopus trochiloides }\end{array}$ & & & & & & $\mathrm{X}$ & & & & \\
\hline $\begin{array}{l}\text { Black-naped Monarch } \\
\text { (Hypothymis azurea) }\end{array}$ & & & & $\mathrm{X}$ & & $\mathrm{X}$ & & $\mathrm{X}$ & & $\mathrm{X}$ \\
\hline $\begin{array}{l}\text { Bronzed Drongo } \\
\quad \text { (Dicrurus aeneus) }\end{array}$ & & & & & & & & & $\mathrm{X}$ & \\
\hline $\begin{array}{l}\text { Velvet-fronted Nuthatch } \\
\text { (Sitta frontalis) }\end{array}$ & & & $\mathrm{X}$ & & & & & & & \\
\hline $\begin{array}{c}\text { Black-headed Cuckoo Shrike } \\
\text { (Lalage - melanoptera) }\end{array}$ & & & & & & & & & $\mathrm{X}$ & \\
\hline
\end{tabular}

The core species that emerge as important are marked with an $\mathrm{X}$ in the corresponding flock richness. Core species from raw networks are marked in yellow.

Table 2 Modularity for different flock species richness classes for raw and filtered networks

\begin{tabular}{lll} 
Flock richness & Raw network & Filtered network \\
\hline 2 species & 0.418 & 0.599 \\
3-5 species & 0.212 & 0.594 \\
$6-10$ species & 0.075 & 0.551 \\
$11-22$ species & 0.015 & 0.071 \\
\hline
\end{tabular}


(a) Raw Network 2 species

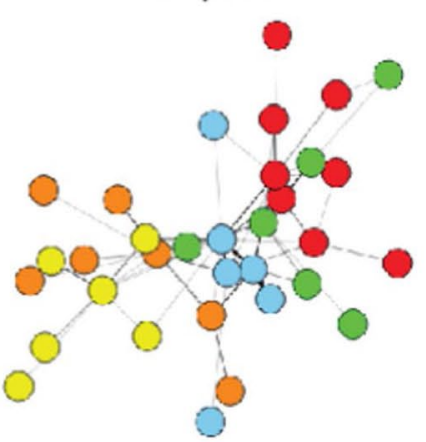

3-5 species

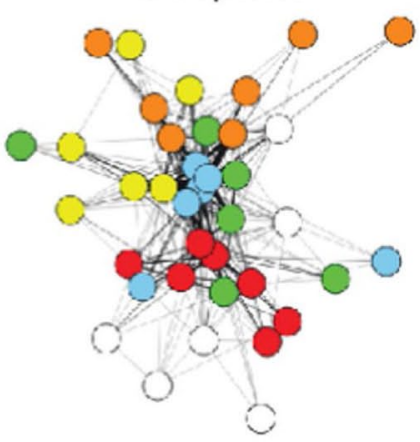

6-10 species

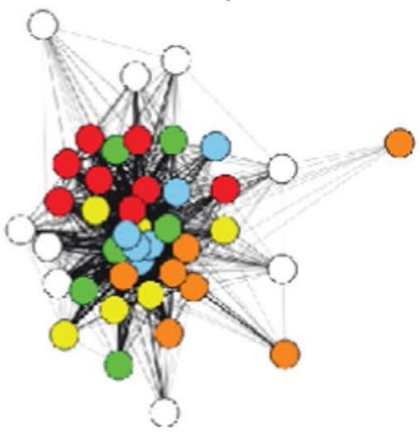

11-22 species

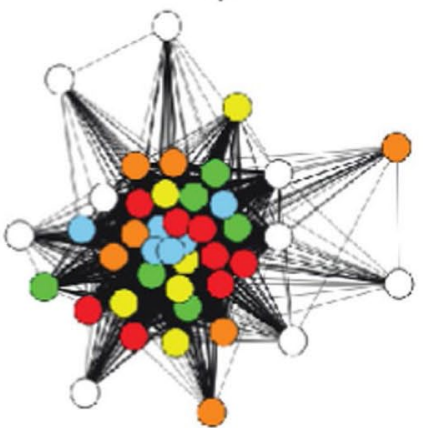

(b) Filtered Network 2 species

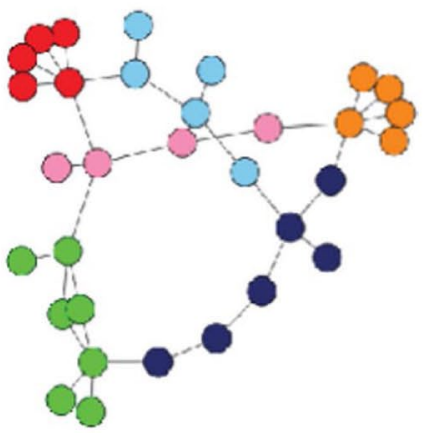

3-5 species

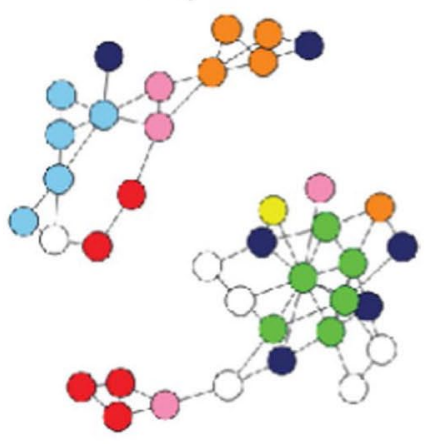

$6-10$ species

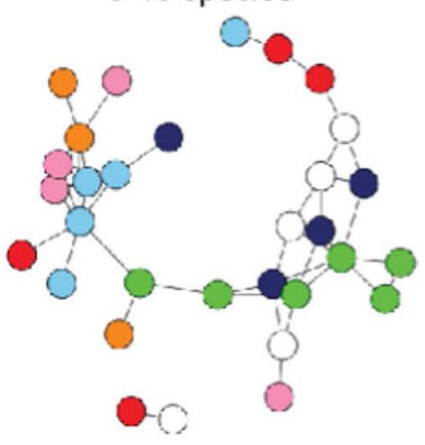

11-22 species

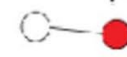

Fig. 1 Communities in raw networks (a) and filtered networks (b) for different flock-richness classes. The communities are detected from the two-species networks and are color coded by the groups identified in the two-species network. We can observe reduced segregation in the larger flock networks. The nodes that do not appear in the two-species flock networks are color coded in white 
(a)

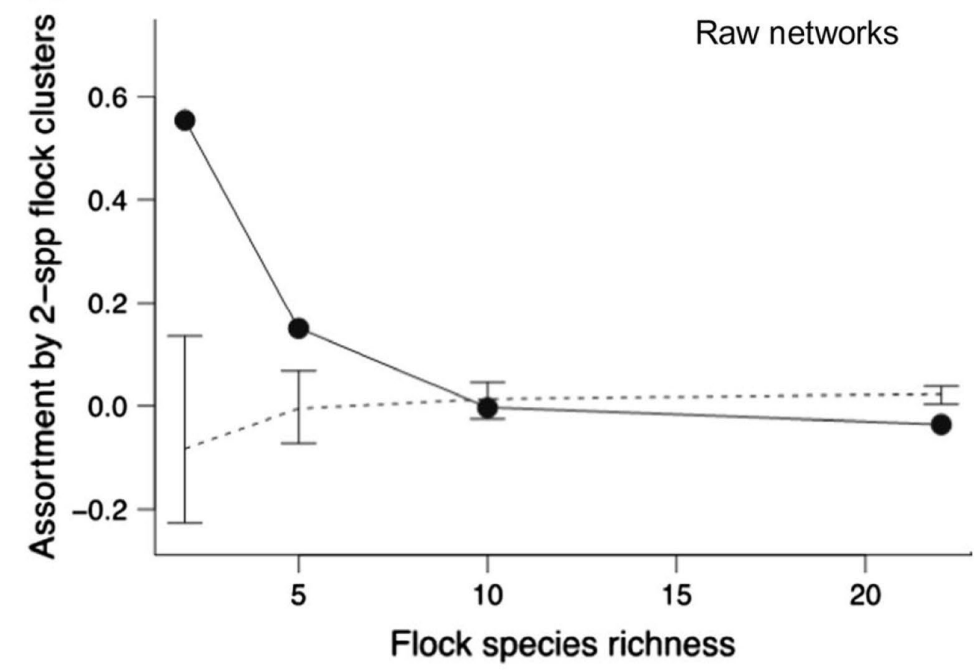

(b)

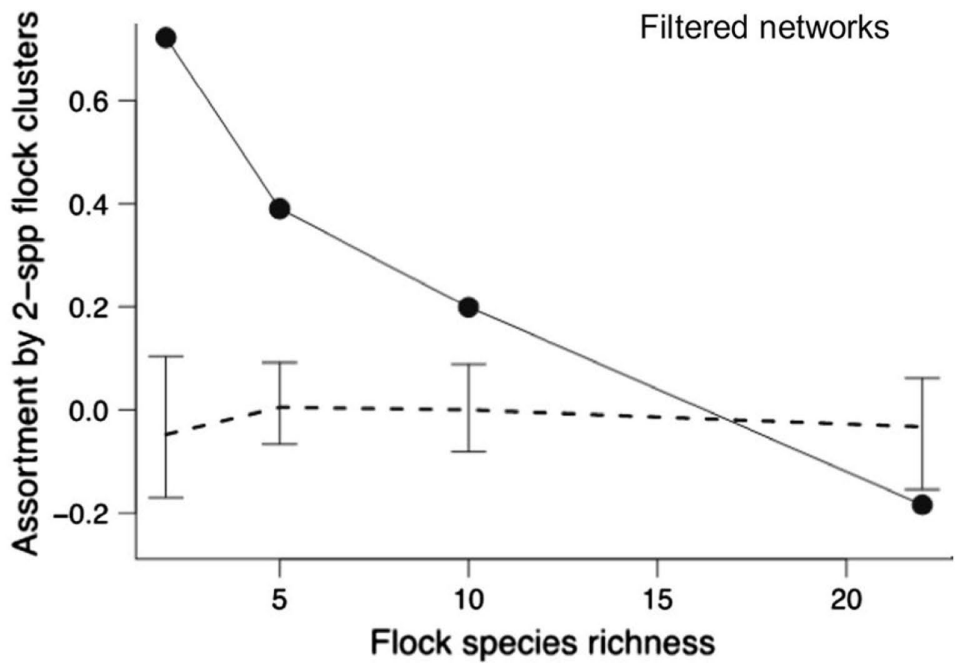

Fig. 2 Relationship between assortativity coefficient (based on two-species flock functional groups) and flock richness in raw networks (a) and filtered networks (b). The bars represent 95\% confidence intervals of association coefficients from 1000 permuted networks, and the dashed lines connect the mean assortativity coefficients from permuted networks from each flock size category. Network visualizations are in Fig. 1 and community assignments in two-species flocks are available in Supplementary material S5 (raw networks) and S6 (filtered networks)

(two-tailed $p<0.001$ ) in 2 species and 3-5 species flocks, is no different from random in 6-10 species flocks, while the relationship becomes negative in 11-22 species flocks (two-tailed $\mathrm{p}<0.001$ ). This suggests that birds that associate strongly in smaller flocks become less likely to associate in larger flocks (Fig. 2a). 
Filtered network

In the filtered network, we found similar clusters as detected in the raw network with higher assortativity overall compared to the raw network (Supplementary material S5, S6, Fig. 2). Just as in the raw networks, the pattern of assortativity by the functional groups identified in two-species flocks again decreases as flocks become larger, and even becomes negative in the largest flocks (Fig. 2b). The species within these functional groups are more assortative than expected (two-tailed $p<0.001$ ) in 2, 3-5 and 6-10 species flocks, while the pattern is negative (two-tailed $p<0.001$ ) in 11-22 species flocks (Fig. 2b).

\section{Discussion}

In this study, we examined species importance and social structure in mixed-flocks in a social network analysis framework. We identified species that emerge as important in networks of varying flock richness. We found that the identity of structurally important species changes with an increase in flock richness. A few species that are important in two-species flocks (where functional importance can more reliably inferred) did not emerge as important in networks built using larger flocks, while some species emerge as structurally important only in larger flocks. In earlier flock network studies (Borah et al. 2018; Mammides et al. 2018; Marthy and Farine 2018; Mokross et al. 2014), structural importance from all-flock networks was interpreted as overall species importance in flocks. However, our study demonstrates that structural importance based on centrality measures from networks built from all flocks may mask variation across different richness levels. We also found that communities detected in small flocks are more assorted (based on communities detected in two-species flock networks) than those in larger flock networks. Ecologically, two-species flocks are very specific associations of species and contain biologically meaningful interactions. In small flocks, these interactions are specifically between certain species and the modules of species associations within these are maintained. More assorted flocks will have clearly separated modules of similar species (where similarity reflects that these species belong to the same module in 
two-species flock networks). From our previous work we know that species in small flocks (2-5 species flocks) are more similar than expected by chance (Bangal et al. 2021). This assortativity decreases systematically as flocks increase in richness, even becoming negative in the largest flocks. Flocks appear to start as smaller subunits of species that associate strongly with each other, and these communities seem to merge in larger flocks.

\section{Important species in flocks of different richness classes}

Among the species in two-species flock networks, some structurally important species continue to remain important in the larger flock networks, while other species emerge as important only in the largeflock networks. In the raw networks, the intraspecifically gregarious species, brown-cheeked fulvetta and western-crowned warbler, are important in two-species networks and they continue to remain important in larger flock networks as they are in the 'All flocks' networks as well. Their presence as core species in two-species flocks indicates a higher functional importance as opposed to species that emerge as important in large flocks. Given that they are intraspecifically gregarious species, which is a functionally important guild in flocks in general (Sridhar and Shanker 2014), and given their structural importance in networks, the functional and structural importance of these species is correlated. Some other species that are important in the two-species flock networks, such as oriental white-eye, dark-fronted babbler and a sallying species, the ashy drongo, do not appear in the core of large flocks. These species may have more functional value in small flocks in comparison to those that are important only in larger flocks (e.g. greenish warbler). Most studies on mixed-species flocks use all flocks to make inferences about flock composition and functioning. However, the patterns we detect in small flocks are different from the all-flock networks. Our study highlights for the first time the need to account for the effect of flock richness on community-level interdependencies.

We found a pattern similar to the raw network for species that emerge as important in the filtered network. The intraspecifically gregarious species and the greater racket-tailed drongo emerge as important in two-species flock networks. In this case, the browncheeked fulvetta emerged important in the 3-5 species flocks and 
remains important in all networks after, while the other species appear inconsistently through the large networks. The greater rackettailed drongo also emerged as important consistently, except in the 3-5 species flocks in the filtered network (Table 1). Species such as the bronzed drongo and the black-headed cuckoo shrike appear as important only in the larger flocks while the velvet-fronted nuthatch emerges in the core of small flocks alone. Some of these species are not known to be functionally important in flocks. However, their importance in the network structure in the filtered network indicates that these species are regular participants in flocks that make a significant contribution to some of the associations in flocks. These associations may be important to hold the network structure and interactions intact and perhaps reflects their importance in the overall bird community that participate in mixed-species flocks.

\section{Network clusters and assortment}

Several distinct clusters were detected from two-species flock networks in both the raw network ( 5 clusters) and filtered networks ( 7 clusters) which reflect how discrete sets of species maintain close associations in small flocks (Bangal et al. 2021). Barring a few differences in clusters detected in the raw vs filtered network, the modules remain similar. We used these clusters as functional groups and examined assortativity by these in larger flock networks; we found that assortativity decreases with increase in flock species richness. The assortativity analysis suggests that two-species flocks, which are the first stage in flock formation, start as very specific well-separated associations. As flock sizes increase, links between these assorted clusters become more common, leading to an overall decrease in separation between clusters detected early on. We observe that species that are strongly associated with each other in small flocks become less likely to be connected in the largest flocks (i.e., 11-22 species). This is in line with our results from our previous work where we found that large flocks tend to be phenotypically more over-dispersed than expected by chance, whereas small flocks are more phenotypically clumped than expected by chance (Bangal et al. 2021). Overall, raw networks have lower assortativity compared to filtered networks of the same flock-richness classes. 
Based on the natural history of the participants, we speculate that the clusters of species that are detected in the two-species flocks could be driven by a few different factors-

1. The vertical stratification of species while foraging e.g. the darkfronted babbler and the white-bellied blue flycatcher are both understory species that cluster together with some other undermid story foragers like the Asian paradise flycatcher;

2. Complementary associations between species where at least one species benefits from a complementary foraging or vigilance habit of the other flocking partner e.g. greater racket-tailed drongo, which is a vigilant species and the common flameback which is a woodpecker that forages on the bark of trees cluster together;

3. Associations where a solitary species benefits from an increased group size by joining a group of intraspecifically gregarious species e.g. ashy drongo a solitary forager) and orange minivet an intraspecifically gregarious species (Refer to S5 and S6 for demonstration of these species clustering together).

It is likely that some of these associations are a combination of multiple factors. However, these factors can lead to the assortment we see in the two-species associations. We found that these clusters start forming more links with the other clusters with increase in flock richness and large flocks show little structure based on the assignments from two-species flocks.

\section{Comparison of raw and filtered networks}

Although the use of all associations in networks can lead to detecting chance associations, we believe that the raw network provides a picture of the group-level dynamics and interactions between species in mixed-species flocks. We reduced the likelihood of chance occurrences by dropping species that are rare in flocks and using the raw network to examine species importance and assortativity. We make the distinction between these and the filtered associations network which, by filtering out the non-significant associations in the dataset, focus on the links that are highly positively correlated. While the raw network captures all of the biologically meaningful group-level 
interactions, we draw inferences from the filtered networks about the interactions that emerge after filtering out the edges that are statistically nonsignificant. Some species, e.g. the velvet-fronted nuthatch, bronzed drongo and the black-headed cuckoo-shrike, are species that emerged as important only in the large flocks, and only in the filtered network. It is likely that these species are not functionally important but participate in flocks regularly, contribute to group augmentation and are key nodes in the cluster. They contribute significantly to the structural integrity of the large flocks and the all- flocks network.

\section{Network structure}

Given the nature of relationships in mixed-species flocks, the under-

lying structure that emerges from specific interdependencies may be masked when examining flocks across the entire spectrum of flock species richness. We suggest that examining patterns of species associations that emerge in flocks of different species richness can provide insight into the relationship between structural and functional importance of species in communities. Many tropical mixed-species flock systems show variation in flock richness (e.g. Chen and Hsieh 2002; Goodale et al. 2009; Graves and Gotelli 1993; Moynihan 1962; Munn 1984; Nimnuan et al. 2004; Sridhar 2013; Srinivasan et al. 2012) and have a few functionally important nuclear species in them. Examining differences in structure of networks built from different species richness also provides insights into how flocks may initiate, and how links may be established between sub-structures for the formation of large flocks.

In particular, integrating the knowledge about functionally important and central species in two-species flock networks is a useful approach to identifying the keystone species in bird communities of which flocks are often an integral part. While two-species flocks may sometimes be transient and may be joined by more species, these associations often last for longer durations and represent the clearest instances where at least one of the two-species benefits from the interaction (Sridhar and Shanker 2014) and are a useful tool to study interdependencies in mixed-species flocks. Our methodological approach can, therefore, also potentially help us understand how flocks assemble and disassemble in areas where flocks are dynamic and show turnover of species over small timescales. 
Network studies on mixed-species flocks have become a popular approach to understanding group-level interactions and also inferring community-level importance of within-trophic keystones in bird communities (Borah et al. 2018; Farine and Milburn 2013; Marthy and Farine 2018; Mokross et al. 2014; Sridhar et al. 2013). The importance of integrating behavioral variation in participation and association of individuals of species in flocks has been emphasized in different studies (Farine and Milburn 2013). However, given the diversity and number of species that participate in mixed-species flocks in many tropical sites and the limitations this imposes on the different methodologies used, the approach of using species-level participation networks is useful. We therefore need both species and individual level network studies, since each of these provide unique insights into community-level interactions between species. In the mixed-species flock context, they provide valuable insights into species interactions and importance in mixed-species flocks on a scale between individual pairwise interactions and overall flock-level properties. Finally, studies on mixed-species groups span different taxonomic groups and are likely to have common underlying principles. Our study provides a framework for understanding interactions and associations for different taxa in multiple contexts.

\section{2}

Author contributions PB developed this project with guidance from HS and KS. PB and HS collected the data for this study. DS and LVM contributed to discussions related to the analysis. PB and DS analyzed this data with inputs from all other authors. PB wrote the first draft of the manuscript. All authors contributed to the following drafts of the manuscript.

Data availability The data used in this study is available as supplementary material.

Supplementary Information Four supplemental files (1 pdf, 3 csv) are attached to the archive record for this document. 


\section{References}

Bangal P, Sridhar H, Shanker K (2021) Phenotypic clumping decreases with flock richness in mixed-species bird flocks. Front Ecol Evol. https://doi.org/10.3389/ fevo.2020.537816

Beauchamp G (2013) Social predation: how group living benefits predators and prey. Elsevier

Borah B, Quader S, Srinivasan U (2018) Responses of interspecific associations in mixed-species bird flocks to selective logging. J Appl Ecol 55(4):1637-1646. https://doi.org/10.1111/1365-2664.13097

Chen CC, Hsieh F (2002) Composition and foraging behaviour of mixed-species flocks led by the Grey-cheeked Fulvetta in Fushan Experimental Forest. Taiwan Ibis 144(2):317-330. https://doi.org/10.1046/j.1474-919X.2002.00020.X

Croft DP, James R, Krause J (2008) Exploring animal social networks. Princeton University Press

Farine DR (2014) Measuring phenotypic assortment in animal social networks: weighted associations are more robust than binary edges. Anim Behav 89:141153. https://doi.org/10.1016/j.anbehav.2014.01.001

Farine DR, Milburn PJ (2013) Social organisation of thornbill-dominated mixedspecies flocks using social network analysis. Behav Ecol Sociobiol 67(2):321330. https://doi.org/10.1007/s00265-012-1452-y

Farine DR, Garroway CJ, Sheldon BC (2012) Social network analysis of mixedspecies flocks: exploring the structure and evolution of interspecific social behaviour. Anim Behav 84(5):1271-1277. https://doi.org/10.1016/j. anbehav.2012.08.008

Franks DW, Ruxton GD, James R (2010) Sampling animal association networks with the gambit of the group. Behav Ecol Sociobiol 64(3):493-503. https://doi. org/10.1007/s00265-009-0865-8

Giraldeau L-A, Beauchamp G (1999) Food exploitation: searching for the optimal joining policy. Trends Ecol Evol 14(3):1017-1021

Girvan M, Newman MEJ (2002) Community structure in social and biological networks. PNAS 99(12):7821-7826. https://doi.org/10.1079/pavsnnr20116044

Goodale E, Nizam BZ, Robin VV, Sridhar H, Trivedi P, Kotagama SW, Vijayan L (2009) Regional variation in the composition and structure of mixed-species bird flocks in the Western Ghats and Sri Lanka. Curr Sci 97(5):648-663

Goodale E, Beauchamp G, Ruxton GD (2017) Mixed-species groups of animals: behaviour, community structure and conservation. Academic Press, London

Goodale E, Sridhar H, Sieving KE, Bangal PZ, Farine DR, Martínez AE (2020) Mixed company : a framework for understanding the composition and organization of mixed-species animal groups. Biol Rev. https://doi.org/10.1111/ brv. 12591

Graves GR, Gotelli NJ (1993) Assembly of avian mixed-species flocks in Amazonia. Proc Natl Acad Sci USA 9o(4):1388-1391. https://doi.org/10.1073/ pnas.90.4.1388 
Greenberg R (2000) Birds of many feathers: the formation and structure of mixed- species flocks of forest birds. In: Boinski S, Garber PA (eds) On the move: how and why animals travel in groups. The University of Chicago Press, Chicago, pp 521-558

Harrison NM, Whitehouse MJ (2011) Mixed-species flocks: an example of niche construction? Anim Behav 81(4):675-682. https://doi.org/10.1016/j. anbehav.2011.01.013

Jullien M, Clobert J (2000) The survival value of flocking in neotropical birds : reality or fiction? Ecology 81(12):3416-3430

Krebs JR (1973) Social learning and the significance of mixed-species flocks of chickadees (Parus spp.). Can J Zool 51(12):1275-1288. https://doi.org/10.1139/ z73-181

Lukoschek V, McCormick MI (2000) A review of multi-species foraging associations in fishes and their ecological significance. Proc Int Coral Reef Sympos 1:23-27

Magrath RD, Pitcher BJ, Gardner JL (2009) An avian eavesdropping network: alarm signal reliability and heterospecific response. Behav Ecol 20(4):745-752. https://doi.org/10.1093/beheco/arpo55

Mammides C, Chen J, Goodale UM, Kotagama SW, Goodale E (2018) Measurement of species associations in mixed-species bird flocks across environmental and human disturbance gradients. Ecosphere. https://doi.org/10.1002/ecs2.2324

Marthy W, Farine DR (2018) The potential impacts of the songbird trade on mixed-species flocking. Biol Conserv 222:222-231. https://doi.org/10.1016/j. biocon.2018.04.015

Mokross K, Ryder TB, Côrtes MC, Wolfe JD, Stouffer PC (2014) Decay of interspecific avian flock networks along a disturbance gradient in Amazonia. Proc R Soc B Biol Sci 281:01-10. https://doi.org/10.1098/rspb.2013.2599

Moynihan M (1962) The organization and probable evolution of some mixed species flocks of neotropical birds. Smithson Misc Collect 143:1-140

Munn CA (1984) The behavioral ecology of mixed-species bird flocks in Amazonian Peru. Princeton University, Princeton

Munn CA, Terborgh JW (1979) Multi-species territoriality in Neotropical foraging flocks. Condor 81(4):338-347. https://doi.org/10.2307/1366956

Newman MEJ (2002) Assortative mixing in networks. Phys Rev Lett 89(20):1-4. https://doi.org/10.1103/PhysRevLett.89.208701

Newman MEJ (2003) Mixing patterns in networks. Phys Rev E 67(2):13. https:// doi.org/10.1103/PhysRevE.67.026126

Newman MEJ (2006) Modularity and community structure in networks. PNAS 103(23):8577-8582. https://doi.org/10.1073/pnas.0601602103

Nimnuan S, Round PD, Gale GA (2004) Structure and composition of mixedspecies insectivorous bird flocks in Khao Yai National Park. Nat Hist b Siam Soc 52:71-79

Shizuka D, Farine DR (2016) Measuring the robustness of network community structure using assortativity. Anim Behav 112:237-246. https://doi. org/10.1016/j.anbehav.2015.12.007 
Sridhar H (2013) Causes and consequences of heterospecific foraging associations in terrestrial bird communities. Indian Institute of Science, Bengaluru

Sridhar H, Shanker K (2014) Importance of intraspecifically gregarious species in a tropical bird community. Oecologia 176(3):763-770. https://doi.org/10.1007/ s00442-014-3045-0

Sridhar H, Beauchamp G, Shanker K (2009) Why do birds participate in mixedspecies foraging flocks? A large-scale synthesis. Anim Behav 78(2):337-347. https://doi.org/10.1016/j.anbehav.2009.05.008

Sridhar H, Jordán F, Shanker K (2013) Species importance in a heterospecific foraging association network. Oikos 122(9):1325-1334. https://doi. org/10.1111/j.1600-0706.2013.00101.x

Srinivasan U, Raza RH, Quader S (2012) Patterns of species participation across multiple mixed-species flock types in a tropical forest in northeastern India. J Nat Hist 46:2749-2762. https://doi.org/10.1080/00222933.2012.717644

Stensland EVA, Angerbjörn A, Berggren PER (2003) Mixed species groups in mammals. Mamm Rev 33(3):205-223

Vasas V, Jordán F (2006) Topological keystone species in ecological interaction networks: considering link quality and non-trophic effects. Ecol Model 196(34):365-378. https://doi.org/10.1016/j.ecolmodel.2006.02.024

Weiss MN, Franks DW, Brent LJN, Ellis S, Silk MJ, Croft DP (2021) Common datastream permutations of animal social network data are not appropriate for hypothesis testing using regression models. Methods Ecol Evol 12(2):255-265. https://doi.org/10.1111/2041-210X.13508

Whitehead H, Dufault S (1999) Techniques for analyzing vertebrate social structure using identified individuals: review and recommendations. Adv Study Behav 28:33-74 